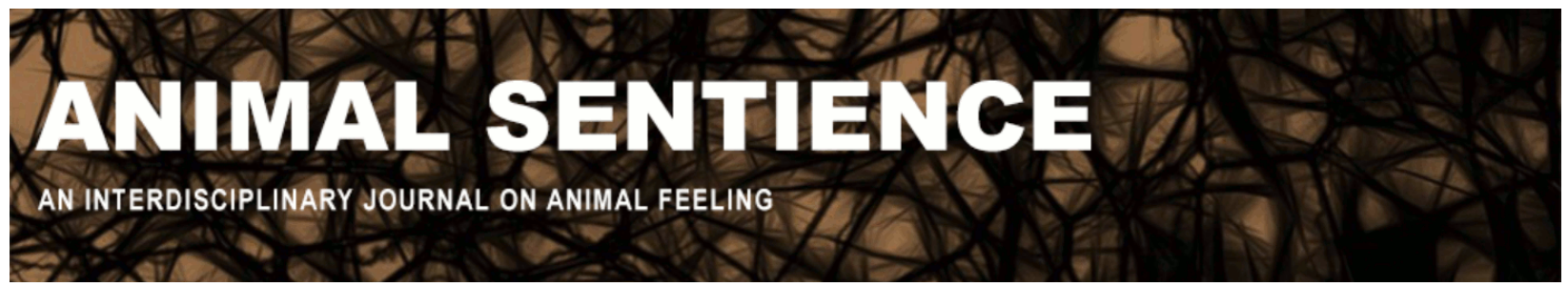

King, Barbara J (2016) Animal mourning: Précis of How animals grieve (King 2013). Animal Sentience 4(1)

DOI: $10.51291 / 2377-7478.1010$

Date of submission: 2015-04-07

Date of acceptance: 2015-12-11

(c)

This article has appeared in the journal Animal

Sentience, a peer-reviewed journal on animal

cognition and feeling. It has been made open access,

free for all, by WellBeing International and deposited

in the WBI Studies Repository. For more information,

please contact

wbisr-info@wellbeingintl.org.

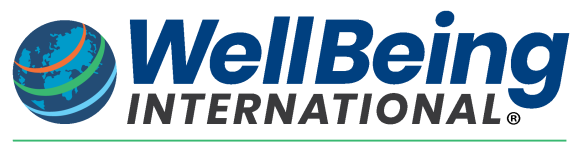

SOLUTIONS FOR PEOPLE, ANIMALS AND ENVIRONMENT 
Multiple Book Review: Animal Sentience publishes multiple reviews of selected books whose Précis is published in the journal along with the reviews and the author's response(s). Most of the multiple book reviews are invited individually by the Editor but freely submitted reviews of the selected books whose Précis has appeared can also be considered for publication. Books can also be nominated and reviewers can be proposed by writing to the Editor.

Instructions: http://animalstudiesrepository.org/animsent/guidelines.html

[Editorial Note: Multiple invited reviews of this book will be appearing in upcoming issues of Animal Sentience, together with the author's Response.]

\title{
Animal mourning
}

Précis of How animals grieve (King 2013)

\author{
Barbara J. King \\ Department of Anthropology \\ College of William \& Mary
}

\begin{abstract}
When an animal dies, that individual's mate, relatives, or friends may express grief. Changes in the survivor's patterns of social behavior, eating, sleeping, and/or of expression of affect are the key criteria for defining grief. Based on this understanding of grief, it is not only big-brained mammals like elephants, apes, and cetaceans who can be said to mourn, but also a wide variety of other animals, including domestic companions like cats, dogs, and rabbits; horses and farm animals; and some birds. With keen attention placed on seeking where grief is found to occur and where it is absent in wild and captive animal populations, scientists and others interested in animal emotion and animal minds can build up a database that answers questions about patterns of grief in the animal kingdom. The expression of grief is expected to be highly variable in individuals within populations, based on an animal's ontogeny, personality, and relationship to the deceased. Human grief may be unique in our species' ability to anticipate death and to consider its meaning across time and space, and yet such hypothesized species-specific features do not imply a more profound emotional experience in humans compared to other animals. This new knowledge of the depth of animals' capacity for grief invites novel exploration of animal-welfare issues including the use of animals in factory farming, entertainment, and biomedicine.
\end{abstract}

Keywords: grief, mourning, love, emotion

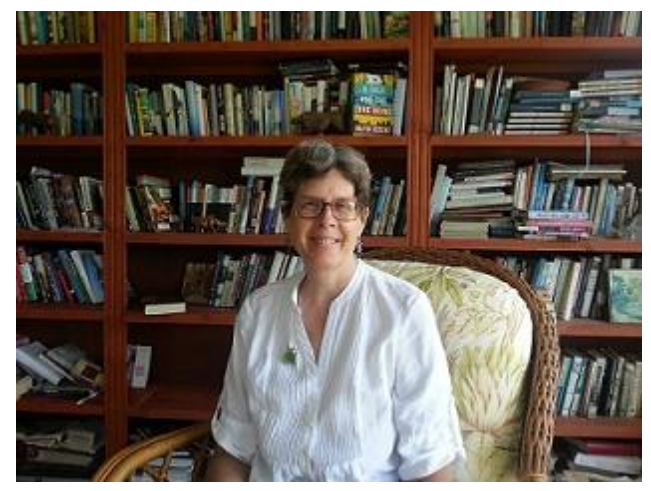

Barbara J. King bjking@wm.edu, Chancellor Professor of Anthropology at the College of William and Mary, writes and speaks about animal thinking, feeling, and welfare. Her article, "When Animals Mourn," in Scientific American was included in the 2014 anthology The Best American Science and Nature Writing. King writes weekly for NPR's 13.7 Cosmos and Culture blog. Her next book, Animals We Eat, is forthcoming. http://www.barbarajking.com 
$\mathbf{T}_{\mathrm{w}}$ wo Mulard ducks, Harper and Kohl, were rescued in 2006 from a foie gras factory in New York and brought to Farm Sanctuary. Frightened of people and suffering from hepatic lipidosis as a result of the force-feeding they had experienced, the birds upon arrival were in bad shape emotionally and physically. Harper was blind in one eye, and Kohl's legs were awry because fractures in them had gone untreated.

As they gradually began to recover at the sanctuary, the two birds became inseparable. For four years, they spent a good deal of time together, to the exclusion of other ducks. Then Kohl's condition began to deteriorate; when he could no longer walk, sanctuary staff prepared to euthanize him. Believing that the opportunity could only help Harper, the staff carried out the procedure in a barn where Harper could see what was going on. When the procedure concluded, Harper at first prodded his motionless friend, but soon lay down next to Kohl and placed his own head and neck over Kohl's. There he stayed, with the body, for some hours.

Harper was never really the same after that. Some days he would venture to the small pond where he had relaxed with Kohl. Again exhibiting heightened nervousness around people, he never chose to bond with another duck. In two months, Harper died.

The goal of How Animals Grieve (hereafter $H A G$ ) was to collect and critically analyze case studies like this one of response to death in animals, some taken from the peer-reviewed science literature and others from reports by observers at sanctuaries, zoos, and homes. Four sets of key conclusions about animal emotions and animal minds serve as a way to summarize $H A G$.

\section{Recognizing the Presence of Grief}

Grief is best defined by comparing the behavior exhibited by survivors after the death of a relative, friend, or mate with the behaviors they exhibited before that death. Significant alterations in patterns of social behavior, eating, sleeping, and/or of expression of emotional affect are the key criteria, and they should persist for hours, days, or weeks. This definition depends on a human observer's familiarity with the daily routines and communicatory signaling of the individual surviving animal. This calls into question the reliability and interpretability of cursory reports (including but not limited to those in the popular media) based on an hour's observation or even just a photograph taken following a death and purported to demonstrate grief. Before-and-after analyses of stress hormones may also be used to measure grief, though $H A G$ takes the position that invasive biological markers must always be analyzed in the context of before-and-after observational data.

This definition, one that focuses on the visible expression of grief measured against a baseline of behavior, helps to counter charges of anthropomorphism that may be brought to bear upon claims of animal emotion and animal thinking. In the case of Harper and Kohl, skeptics might point out that we don't have, and indeed could not possibly have, evidence that Harper understands the finality of what happened to his friend. Couldn't Harper's social withdrawal be explained by the general changes in his day-to-day routine? Perhaps 
he felt disrupted and distressed, but how would we ever know for sure if that disruption and distress was connected to Kohl's death specifically?

Questions like these remind us that we should always seek and give consideration to alternative hypotheses to felt grief (a point that will be taken up again below). The approach in $H A G$, however, is based on two principles. First, cognitive awareness of death and its finality, for example a capacity for distinguishing long-term separation from death, need not be a prerequisite for mourning. Second, we should avoid invoking a priori a double standard when shifting from a consideration of human grief to the grief of other animals. When observing or interacting with humans who exhibit new signs of depression or distress in the wake of the death of a relative, mate, or friend with whom they spent time and with whom they expressed affection or love, we accept that the most likely explanation involves grief. On occasion, that interpretation may be wrong, because for a host of reasons both cultural and individual, not every death evokes feelings of loss and mourning in surviving humans. HAG takes the position, though, that when applied to sentient beings in intimate social connection, the grief interpretation is unlikely to be wrong very often and, on balance, will yield more benefits (including new hypotheses to test) than costs.

\section{Recognizing the Absence of Grief}

Research carried out for $H A G$ uncovered credible instances of grief in animals as diverse as elephants, chimpanzees, dolphins, house cats, rabbits, dogs, and birds. The degree to which the available evidence fit $H A G$ 's definition varied, and so in turn, the strength of the conclusion that grief exists also varies. Elephants are at present the gold standard in animal grief studies, in part because two long-term elephant-behavior projects in Kenya furnished us with examples. At Samburu National Park, for instance, when the elephant matriarch Eleanor was dying, and then when she died, elephants of five different families including her own carried out behaviors of concern or distress, ranging from attempts to right her collapse to rocking in distress over her body.

As noted earlier, alternative hypotheses should be considered and part of the goal of $H A G$ was to determine when expressed grief was absent. Corpse-carrying by mothers when their infants have died is a practice found among nonhuman primates, including chimpanzees and baboons, and cetaceans, where the behavior may include keeping infants afloat in the water or carrying them on one's back. In some cases, the mother significantly altered her routine in ways consonant with $H A G$ 's definition of grief, but in other cases, it appeared that she did not. Among the described cases of infant corpse-carrying, no convincing evidence exists that baboons in Ethiopia or Botswana populations expressed grief: these females continued their daily routines even while holding near-mummified bodies of their infants with one arm. A gelada baboon at Guassa, Ethiopia, proceeded to mate while holding her dead infant. The conclusion in $H A G$ is not that that the female felt no grief, but rather that the female's behavior falls outside the definition of grief. Especially in the wild, natural selection may work against the visible expression of grief, since any of the alterations to an animal's routine may make the animal more vulnerable to predation or other lethal aggression. 
It's too early in the study of animal grief to understand patterns of grief well enough to predict (by species, population, or individual) when it is or is not likely to occur. Right now we have broad predictions, such as these two: intensely social species will tend toward grief responses more than largely solitary ones. Individuals in species with a capacity for grief will exhibit that grief according to a complex mix of ontogeny and personality as well as the nature of the relationship with the deceased. Because the field of animal thanatology is just beginning, scientists and others interested in animal emotion are encouraged to record (on film, ideally, over days) responses of diverse animals living in diverse habitats to naturallyoccurring deaths of relatives, friends, or mates.

\section{How Is Human Mourning Different?}

Even while avoiding the unwarranted excesses of a perspective steeped in human exceptionalism, $H A G$ aims to suggest features of grief that may be unique to humans. Hypotheses about human uniqueness are ripe for falsification by observational animalbehavior research in the future, and at the same time, it makes good evolutionary sense to expect unique aspects of human grief. Elephants may show a generalized response to grief beyond the immediate family - as we saw from Samburu when five different families responded to Eleanor's dying and death - but in HAG there is no report of animals' awareness of or response to death beyond the immediate time and space.

By contrast, humans mourn others of our kind whom we have never met personally and who may live in distant nations or who were alive during other time periods. We visit memorials in Kigali; Rwanda; Berlin, Germany; or New York City, or read the news about killings in Baghdad, Mexico City, or Los Angeles, and we may feel moved to tears; it's not just abstract concepts like genocide, war, terrorism, racism, and other acts of violence that move us, but empathy for the suffering of individual people and their families.

In short, our mourning practices are (to varying degrees based on cross-cultural access to global communication) untethered from time and space. Our mourning rituals, including burial rituals, are comparatively elaborated in symbolic ways, not because we are the only cultural animals, but because our culture developed along a trajectory that puts a premium on community-, language- and technology-based responses to death. HAG traces briefly some of the archaeologically visible highlights of this prehistoric trajectory, noting the nature of intentional burials with grave goods at Neanderthal sites such as La Ferrassie in France and Teshik-Tash in Uzbekistan, and anatomically-modern human sites such as Skhul and Qafzeh in Israel and Sunghir in Russia.

A search for behaviors that evolved newly in the hominin lineage does not imply any expectation that human species-specific grieving practices are more emotionally profound than those of other animals. In the end, what stands out in $H A G$ is not the hypotheses of human uniqueness, but the discovery that other animals do grieve, and that they grieve because they have loved. 


\section{Why Grief Studies Matter for How We Treat Animals}

Explicitly and implicitly, $H A G$ takes up the question of how a deeper understanding of animal love and grief may enable us to work more effectively for animal welfare. Allowing surviving individuals quiet time with the bodies of their relatives, mates, or friends who have just died - as Harper was allowed immediately after Kohl's death - is a practice that is increasingly adopted by people in sanctuaries, small farms, zoos, veterinary practices, and indeed in private homes. We may offer grieving animals the compassion and dignity they deserve as they choose whether to visibly express any emotions they may feel about their loss.

As the grief stories unfold in $H A G$, readers may find themselves thinking anew about the way our society treats animals: about the cows on factory farms who are forcibly and repeatedly separated from their calves, some of those calves taken to slaughter; about the whales and dolphins captured for consumption or entertainment theme parks, either slaughtered in sight of their families or separated from those families; about elephants or lions killed by poachers in East Africa or made to perform in American circuses; and about apes and monkeys kept in U.S. laboratories and forced to undergo invasive biomedical procedures or maternal-deprivation experiments, sometimes ending in death. The rippling effects of these traumas are extensive and may involve grief reactions in numerous animals even beyond the suffering of those individuals most directly targeted.

Opening our minds to the love and grief experienced by other animals may become a source of comfort in our own lives: it is on this reflective note that $H A G$ concludes. In concert with many other books, articles, and essays, HAG asks all of us to think about the consequences of the knowledge that we share the planet with other animals who experience joys and sorrows that stem from intimate connections with others. That knowledge may become action when, on a local scale, we help our grieving animal companions and when, on a global scale, we work to reduce anthropogenic acts that bring grief to other animals in captivity and in the wild.

\section{References}

King, B. J. (2013). How animals grieve. University of Chicago Press.

Editorial Note: Multiple invited reviews of this book will be appearing in upcoming issues of Animal Sentience, together with the author's Response.]

Multiple Book Review: Animal Sentience publishes multiple reviews of selected books whose Précis is published in the journal along with the reviews and the author's response(s). Most of the multiple book reviews are invited individually by the Editor but freely submitted reviews of the selected books whose Précis has appeared can also be considered for publication. Books can also be nominated and reviewers can be proposed by writing to the Editor.

Instructions: http://animalstudiesrepository.org/animsent/guidelines.html 
The Israel Journal of Ecology and Evolution is seeking manuscript submissions for a special edition on Compassionate Conservation. Guest editors are Drs. Dror Ben-Ami and Daniel Ramp. They are inviting original research that is exemplary of Compassionate Conservation values. Compassionate Conservation builds the welfare of individual wildlife into conservation practice to improve outcomes for individuals, species and ecosystems. It has four guiding principles: a commitment to doing no harm as a starting point for intervention, individuals matter, all wildlife are worthy of conservation, and peaceful coexistence should be the ultimate aim for conservation practice. Practical examples include non-lethal management of wildlife populations, including animal welfare in conservation solutions, non-invasive monitoring techniques for studying wildlife, and engagement of human communities to minimise human-wildlife conflict.

For more information please contact Daniel Ramp at Daniel.Ramp@uts.edu.au 